\title{
Interleukin-8 Induces the Endothelial Cell Migration through the Activation of Phosphoinositide 3-Kinase-Racl/RhoA Pathway
}

\author{
Yi Lai 1,2,", Yang Shen 1,2, ${ }^{*}$ Xiao-Heng Liu 1,2 ${ }^{凶}$, Yi Zhang ${ }^{3}$, Ye Zeng 1,2, Ying-Fen Liu ${ }^{3}$ \\ 1. Laboratory of Cardiovascular Diseases, West China Hospital, Sichuan University, Chengdu 610041, China \\ 2. Institute of Biomedical Engineering, West China School of Preclinical and Forensic Medicine, Sichuan University, \\ Chengdu 610041, China \\ 3. Laboratory of Biomedical Ultrasonics, West China Second University Hospital, Sichuan University, Chengdu 610041, \\ China \\ * These authors contributed equally to this work.
}

$\triangle$ Corresponding author: Laboratory of Cardiovascular Diseases, West China Hospital, Sichuan University, No.17 Renmin Nanlu 3 Duan, Chengdu, 610041, China. E-mail: liuxiaohg@scu.edu.cn; Tel: 0086-28-85503400; Fax: 0086-28-85503400.

(C) Ivyspring International Publisher. This is an open-access article distributed under the terms of the Creative Commons License (http://creativecommons.org/ licenses/by-nc-nd/3.0/). Reproduction is permitted for personal, noncommercial use, provided that the article is in whole, unmodified, and properly cited.

Received: 2011.02.11; Accepted: 2011.06.01; Published: 2011.06.21

\begin{abstract}
Endothelial cell migration is essential for tumor angiogenesis, and interleukin-8 (IL-8) has been shown to play an important role in tumor growth, angiogenesis, and metastasis. This study aimed to investigate the molecular mechanism of IL-8 induced endothelial cell migration. Our results indicated that IL-8 induced a rapid rearrangement of the actin cytoskeleton in EA.Hy926 cells, generating extensions resembling membrane ruffling and stress fibers. These processes required parallel upregulation of the small GTPases Rac1 and RhoA. Moreover, we demonstrated that IL-8 activated PI3K following the same kinetics observed from IL-8 induction of cytoskeletal rearrangement, suggesting the participation of PI3K in these processes. Taken together, our study demonstrates that PI3K-Rac1/RhoA signaling pathway plays a vital role in IL-8 induced endothelial cell migration, and provides new insight into the molecular mechanisms by which IL- 8 contributes to tumor angiogenesis and metastasis.
\end{abstract}

Key words: Rac1; Interleukin-8; PI3-kinase; cell migration; tumor angiogenesis

\section{Introduction}

As an inflammatory and chemotaxis factor, interleukin-8 (IL-8) belongs to the CXC family of proteins and is readily purified from human monocyte culture supernatant stimulated with lipopolysaccharide (LPS) or phytohemagglutinin (PHA). IL-8 was discovered by Yoshimura [1] in 1987 and is mainly secreted by monocytes and endothelial cells. IL-8 activates multiple intracellular signaling pathways downstream of two cell-surface receptors, CXCR1 and CXCR2. Increased expression of IL-8 and/or its re- ceptors has been characterized in cancer cells, endothelial cells, infiltrating neutrophils, and tumor-associated macrophages, indicating that IL-8 may function as a regulatory factor in the tumor microenvironment. Tumor-derived IL-8 has the capacity to exert profound effects on the tumor microenvironment. For example, IL-8 secretion can activate endothelial cells in the tumor vasculature to promote angiogenesis. The multiple effects of IL-8 on different cell types present within the tumor microenvironment 
suggest that targeting of IL-8 signaling may have important implications for sensitizing tumors to chemotherapeutic and biological agents.

The small G protein Rac1 is one of the main regulatory factors involved in the reassembly of the actin cytoskeleton, which plays important role in coordinating cell migration [2]. It has been found that external stimulus could be transduced through matrix-integrin-Rho GTPases signal pathway, suggesting that Rac1 may be one of the critical molecules involved in IL-8 induced endothelial cell migration. On the other hand, phosphoinositide 3-kinases (PI3Ks) function in maintaining cell polarity and defining the leading edge of migrating cells [3]. The feedback loops between Rho GTPases and PI3K can integrate and amplify cell signaling, and both of them are required for efficient cell migration.

Our previous study demonstrated that IL-8 could induce endothelial cell migration [4]. In addition, IL-8 induced the expression of Rac1, which then modulated cell migration [5]. However, whether PI3K is involved in IL-8 induced endothelial cell migration remains largely unexplored. Therefore, the present study focused on the link between PI3K and Rac1/RhoA downstream of IL-8 in mediating endothelial cell migration. The results demonstrated that PI3K-Rac1/RhoA signaling pathway plays a vital role in the IL-8 induced endothelial cell migration.

\section{Materials and methods}

\section{Cell culture}

The EA.Hy926 cell line (Blood Research Institute, Jiangsu Province, China) is a hybridoma between human umbilical vein endothelial cells (HUVECs) and the epithelial lung tumor cell line A549 [6]. This cell line retains most features of HUVECs, including the expression of endothelial adhesion molecules and human factor VIII-related Ag [7]. EA.Hy926 cells were grown to confluence in Dulbecco's modified Eagle's medium (DMEM) supplemented with 5\% fetal bovine serum (PromoCell, Heidelberg, Germany).

\section{Antibodies and pharmacological reagents}

RhoA monoclonal antibody and peroxidase conjugated goat anti-mouse secondary $\mathrm{mAb}$ were purchased from Santa Cruz Biotechnology (Santa Cruz, CA, USA). Rac1 monoclonal mAb was purchased from Cytoskeleton (Cytoskeleton, Denver, CO, USA). IL-8 was purchased from PeproTech (PeproTech, Rocky Hill, NJ, USA). BODIPY FL phallacidin was obtained from Invitrogen (Invitrogen, Carlsbad, CA, USA). DAPI was purchased from Sigma (Sigma,
St. Louis, MO, USA). Wortmannin was obtained from Calbiochem (Calbiochem, San Diego, CA, USA).

\section{Western blot analysis}

Cell lysate was prepared following the standard procedure and protein concentrations were determined using the Bio-Rad system (Hercules, CA, USA). The lysate was mixed with Laemelli sample buffer, boiled at $100^{\circ} \mathrm{C}$ for $5 \mathrm{~min}$, and then separated by $15 \%$ SDS-acrylamide electrophoresis and transferred to polyvinylidene fluoride (PVDF) membranes (Millipore, Billerica, MA, USA). The membranes were blocked overnight in PBS with 5\% nonfat dried milk and $0.1 \%$ Tween-20, then incubated with the relevant primary and secondary antibodies in blocking buffer for $1 \mathrm{~h}$ and washed three times in PBS plus $0.1 \%$ Tween-20 after the incubation. Blots were developed using the Super-Signal detection system (Pierce, Rockford, IL, USA), exposed to Hyperfilm (Amersham, Arlington Heights, IL, USA) and quantified with quantity one software. Antibodies were diluted as follows: 1:200 for anti-RhoA monoclonal $\mathrm{mAb}$, 1:500 for anti-Rac monoclonal $\mathrm{mAb}$, and 1:4000 for peroxidase conjugated goat anti-mouse secondary $\mathrm{mAb}$.

\section{Scratch-wound migration assays}

EA.Hy926 cells were grown to $80-90 \%$ confluence in a 6-well plate (Corning, NY, USA), and incubated in medium containing 1\% FBS overnight. EA.Hy926 monolayers were scratched with a $200 \mu \mathrm{L}$ pipette tip (Fisher Scientific, Suwanee, GA, USA), washed to remove debris and cultured with medium containing 5\% FBS to facilitate cell migration. Cells were pretreated with wortmannin $(100 \mathrm{nM})$ for $20 \mathrm{~min}$ and then IL-8 $(100 \mathrm{ng} / \mathrm{ml})$ was added and incubated for $6 \mathrm{~h}$. Images were collected at the indicated time points using a $4 \times$ objective on an Olympus microscope (Olympus, Tokyo, Japan).

EA.Hy926 cell migration during the wound repair was analyzed using the image analysis software, Image Pro Plus 6.0 (Media Cybernetics). The acquired images were converted from pixels to micrometers with the use of a calibration image. For each experiment, 10 cells were randomly chosen along each edge of the wound, and cell migration distance at the end of each recording period was computed by

$$
D_{n}=L_{n}-L_{0}
$$

Where $D_{n}$ and $L_{n}$ are the net cell migration distance and the cell position at the metering point $n(h)$, respectively. $L_{0}$ is the original position. 


\section{Immunofluorescent staining}

Cells were plated on collagen I-coated coverslips for $24 \mathrm{~h}$ before the experiment. After incubation in the absence or presence of IL-8, cells were rapidly fixed and permeabilized by immersion in $3.7 \%$ paraformaldehyde, $0.1 \%$ glutaraldehyde, and $0.15 \% \mathrm{mg} / \mathrm{ml}$ saponin fix buffer at $37^{\circ} \mathrm{C}$ for $1 \mathrm{~h}$. The fixed cells were then incubated with BODIPY FL phallacidin (Invitrogen, Carlsbad, CA, USA) and DAPI for $30 \mathrm{~min}$ and then mounted in 50\% glycerol-PBS containing 6 $\mathrm{mg} / \mathrm{ml}$ of $\mathrm{N}$-propyl gallate. All fluorescent images were obtained using laser scanning confocal microscope (Leica TCS SP5, Germany).

\section{Plasmids}

The expression plasmids for activated mutant of Rac1 (pcDNA3-EGFP-Rac1-Q61L) and the dominant negative form of Rac1 (pcDNA3-EGFP-Rac1-T17N) were purchased from Addgene (Boston, MA, USA) [8]. The expression plasmids for activated mutant of RhoA (RhoA63L) and the dominant negative form of RhoA (RhoA188A) were kindly provided by Prof. Chen Yong-Chang (Jiangsu University, China).

\section{Transient transfection}

EA.Hy926 cells were transfected with plasmids using Lipofectamine 2000 (Invitrogen) and OptiMEM medium (Gibco BRL) in the absence of antibiotics according to the manufacturer's instructions. After transient transfection, the mRNA expression of Rac1 and RhoA was detected by RT-PCR. The transfection efficiency was measured by a fluorescence microscope. Transfected cells were passaged at 56-60 h and used after $72 \mathrm{~h}$.

\section{Transwell migration assay}

Transwell ( $8 \mu \mathrm{m}$, Corning, NY, USA) filters were equilibrated in serum containing 1640 medium for $2 \mathrm{~h}$. The 1640 medium containing 10\% FBS was added into the lower chamber of the migration filters. Cells were plated in a volume of $1.5 \mathrm{ml}$ serum-free DMEM per Transwell filter with a density of $1 \times 10^{6}$. Cells were allowed to migrate in $5 \% \mathrm{CO}_{2}$ at $37^{\circ} \mathrm{C}$ for $6 \mathrm{~h}$ and were subsequently fixed by immersion of the filters in methanol at room temperature for $15 \mathrm{~min}$. Filters were washed with deionized water and stained in $0.2 \%$ crystal violet in a $20 \%$ methanol/water solution for 10 min. Cells were removed from the upper surface of the membrane with a cotton swab. Cells that had migrated to the underside of the membrane were counted at $200 \times$ magnification from five random fields on each membrane.

\section{Statistical analysis}

Data were presented as mean \pm SD from at least 10 images obtained from three independent experiments, unless otherwise indicated. Statistical analysis was performed by one-way ANOVA test using SPSS 11.5 software package. Differences were considered significant if $P<0.05$.

\section{Results}

\section{IL-8 promotes the migration of endothelial cells}

Figure 1 showed the migration distance of the cells after stimulated by IL-8 at $100 \mathrm{ng} / \mathrm{ml}$ concentration. The cells migration distance was increased with the duration for both of the IL-8 stimulated group and the control group (Figure 1A). The migration distance of IL-8 stimulated group (at $2 \mathrm{~h}, 4 \mathrm{~h}$, and $6 \mathrm{~h}$ ) were longer than that of the control group (Figure 1B, $P<0.05)$. These results suggested that IL-8 enhances the migration of EA.Hy926 cells.

\section{IL-8 modulates the actin cytoskeleton reorgani- zation in endothelial cells}

We examined the cytoskeletal changes after cells were stimulated by IL- 8 . Figure 2 showed that the cytoskeleton changes occurred at different time points when the cells were stimulated by IL- 8 . In unstimulated serum starved cells (control group), actin filaments were well organized and distributed throughout the cells. However, after stimulated by IL-8, prominent stress fibers appeared in the cells, and one kind of F-actin rich spike-like filopodia, a phenotype associated with active Cdc42, was observed ( $1 \mathrm{~min}$ to $10 \mathrm{~min}$ ). In response to IL- 8 , membrane ruffles formed in the 5 min group and 10 min group. Both in $15 \mathrm{~min}$ group and $30 \mathrm{~min}$ group, lamellipodium could be found at the leading edge of migrating cells. These results indicated that IL-8 induces the actin filaments reorganization which then forms cell-motility structures at the leading edge of the cells. Note the prominent induction of stress fibers from $2 \mathrm{~h}$ to $6 \mathrm{~h}$. Taken together, these data suggest that IL-8 not only induces the membrane ruffling and lamellipodial extensions, but also promotes the formation of actin fibers.

\section{Upregulation of Rac1 and RhoA in the endothelial cells stimulated by IL- 8}

The expression of Rac1 and RhoA in endothelial cells was detected by Western blotting when stimulated by IL-8 for different durations (Figure 3). The results showed that the expression of Rac1 increased rapidly (in $5 \mathrm{~min}$ ), and was distinctive with the control group. Then Rac1 expression decreased (from 10 min to $2 \mathrm{~h}$ ). However, Rac expression increased again 
and reached to about 2-fold of the control group at the 4 to $6 \mathrm{~h}$ duration (Figure 3A). These results indicated that IL-8 could induce the upregulation of Rac1.

Compared with Rac1, the expression of RhoA changed more slowly. There was no significant change in RhoA expression compared to the control group from 0 to $15 \mathrm{~min}$, but RhoA expression increased significantly in $30 \mathrm{~min}$ and $1 \mathrm{~h}$ duration groups $(P<0.05)$ (Figure 3B). Later on, RhoA expression decreased to the level of control group.
A
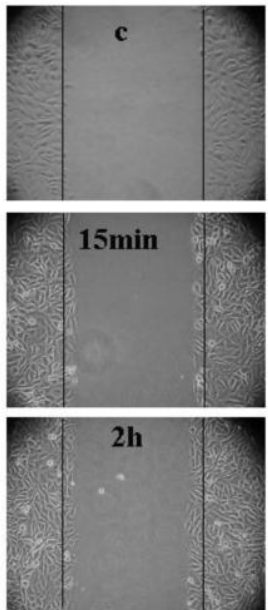
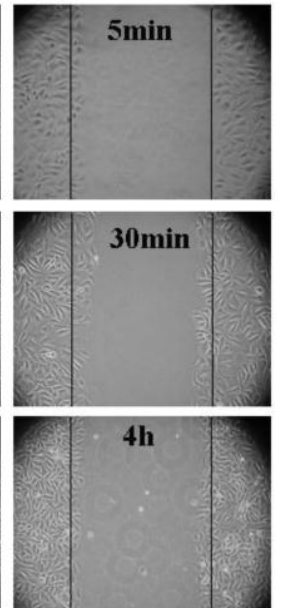
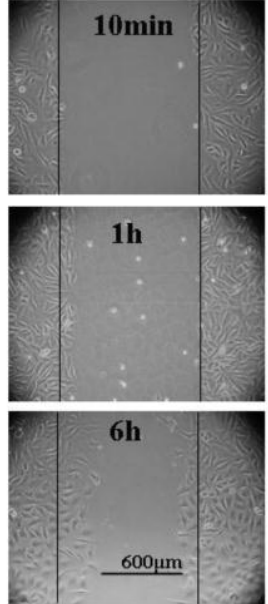

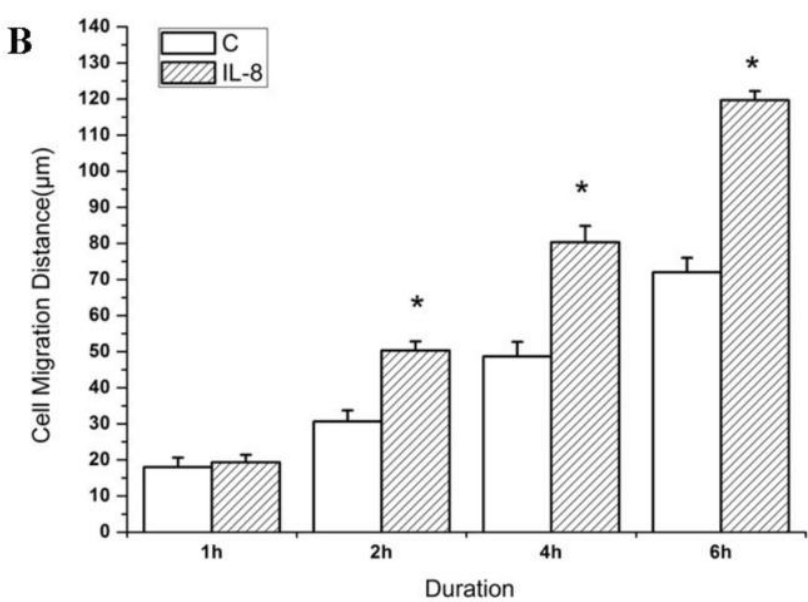

FIG.1. IL-8 induces EA.Hy926 cell migration. (A) Wound healing assay. EA.Hy926 cells were grown to $80-90 \%$ confluence in a 6-well plate, and incubated in medium containing $1 \%$ FBS overnight. EA.Hy926 monolayers were scratched and cultured in medium containing $5 \%$ FBS to facilitate cell migration. Cells were treated with $5 \%$ FBS medium alone (Control), or $100 \mathrm{ng} / \mathrm{mL} \mathrm{IL-8} \mathrm{(IL-8} \mathrm{group).} \mathrm{Cell} \mathrm{migration} \mathrm{was} \mathrm{recorded} \mathrm{by} \mathrm{phase} \mathrm{contrast} \mathrm{microscopy}$ over $6 \mathrm{~h}$ time course after wound scratch. Shown were representative images of wound healing from 0 to $6 \mathrm{~h}$ after wound scratch. (B) The level of cell migration into the wound scratch was quantified as the wound healing distance and compared to that of control cells at each time point. ${ }^{*} P<0.05$ vs. control group. Values represented averages $\pm \mathrm{SE}$ of three independent measurements along the wound scratch. Scale bar $=600 \mu \mathrm{m}$.

FIG.2. IL-8 induces the actin cytoskeleton reorganization. EA. Hy26 cells grown on glass coverslips were fixed with cold methanol and then immunostained with BODIPY FL phallacidin (green) and DAPI (blue). Filopodia were observed at the leading edge of migrating cells (from $1 \mathrm{~min}$ to $10 \mathrm{~min}$ ). Cells showing membrane ruffles were indicated from $5 \mathrm{~min}$ group to $10 \mathrm{~min}$ group. Lamellipodia at the leading edge was showed from $15 \mathrm{~min}$ to $30 \mathrm{~min}$. Note the prominent induction of stress fibers from $2 \mathrm{~h}$ to $6 \mathrm{~h}$. Scale bar $=25 \mu \mathrm{m}$. Shown were representative images of an interphase cell.
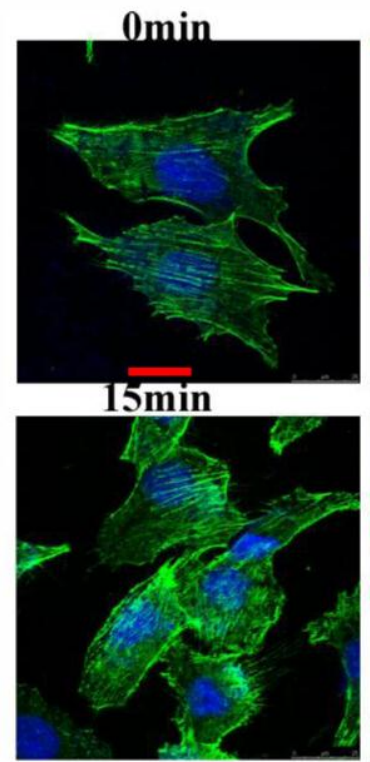

$2 \mathrm{~h}$

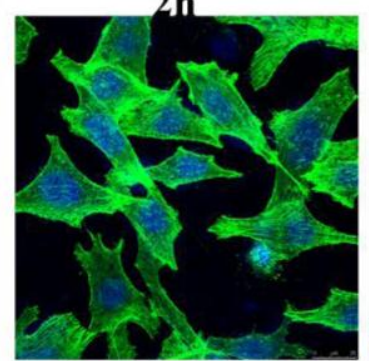

$5 \mathrm{~min}$

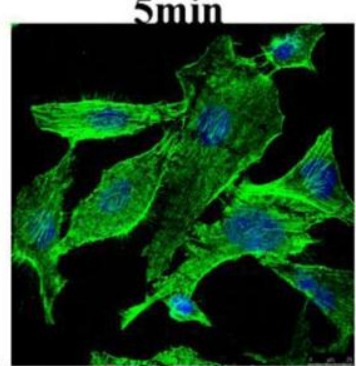

$30 \mathrm{~min}$

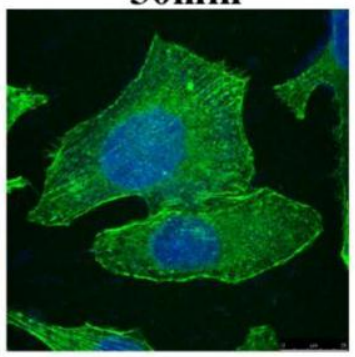

4h

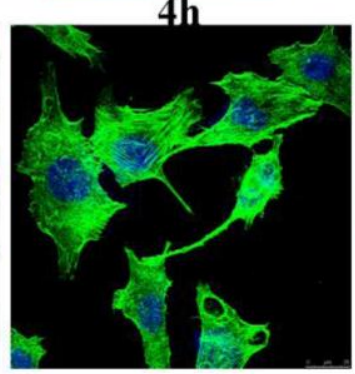

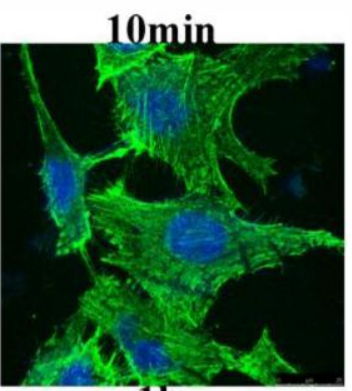

$1 \mathrm{~h}$

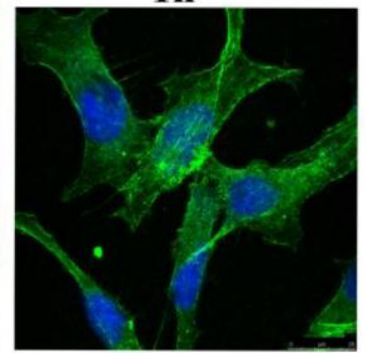

$6 \mathrm{~h}$

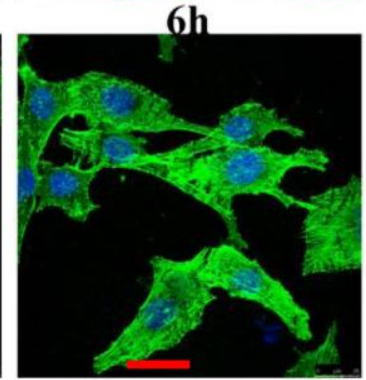



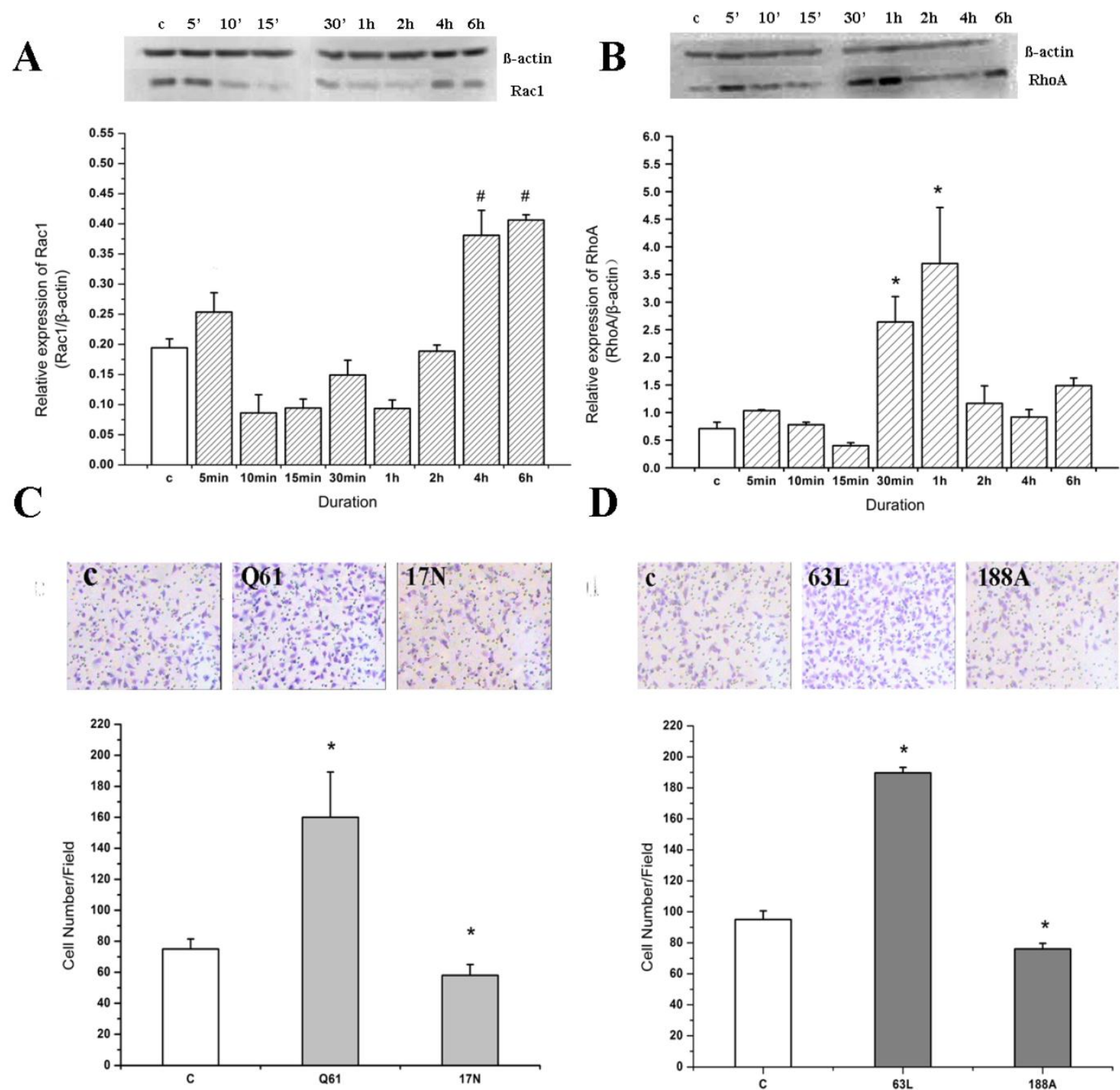

FIG.3. Rac1 and RhoA play important roles in the cell migration induced by IL-8. EA.Hy 926 cells were incubated with $100 \mathrm{ng} / \mathrm{ml} \mathrm{IL}-8$ for $6 \mathrm{~h}$, and then subjected to Western blot analysis using indicated antibodies. B-actin served as loading control. (A) Rac1 expression in $4 \mathrm{~h}$ and $6 \mathrm{~h}$ group was significant different from other groups, ${ }^{\#} P<0.05$; (B) RhoA expression in 30 min group and $1 \mathrm{~h}$ group was significant different from other groups. ${ }^{*} P<0.05$. (C) Cells were transiently transfected with pcDNA3-EGFP-Rac1-Q61L $(10 \mu \mathrm{g})$ or pcDNA3-EGFP-Rac1-T17N $(4 \mu \mathrm{g})$. Forty-eight hours after transfection, cells were examined for cell migration in 6 -well plates with Transwell chambers. $P<0.05$ vs. control group; (D) Cells were transiently transfected with RhoA63L (4 $\mu \mathrm{g})$ or RhoA188A $(6 \mu \mathrm{g})$ and cell migration assay of transfected cell was performed as in (C), ${ }^{*} P<0.05$ vs. control group. 


\section{Rac1 and RhoA are essential for cell migration stimulated by IL-8}

We transfected cells with dominant negative (DN) forms of RhoA or Rac1 and found that DN-RhoA and DN-Rac1 decreased IL-8 induced cell migration significantly (Figure 3C and Figure 3D).

On the other hand, compared with the control group (addition of Lipofectamine only), the amount of migrating cells in the pcDNA3-EGFP-Rac1-Q61L (activated mutant of Rac1) transfection group increased significantly $\quad(P<0.05)$; while that in the pcDNA3-EGFP-Rac1-T17N (DN-Rac1) transfection group was decreased (Figure 3C). Consistent with these results, overexpression of Rac1 significantly enhanced cell migration induced by IL-8, and IL-8 was unable to further increase cell migration in those cells transfected with DN-Rac1. These results suggest that Rac1 might play an important role in IL-8 induced migration of endothelial cells.

Following IL-8 stimulation, the cell migration ability of the RhoA63L (activated mutant of RhoA) transfection group was higher than that of the other groups $(P<0.05)$, while the cell migration ability of the RhoA188A (DN-RhoA) transfection group was inhibited $(P<0.05)$ (Figure 3D). These results indicate that RhoA also plays an important role in IL-8 induced migration of endothelial cells.

\section{PI3K inhibitor inhibits the cell migration induce by IL-8}

To examine whether PI3K is involved in IL-8 induced migration of endothelial cells, we treated the cells with wortmannin (a PI3K inhibitor) and found that wortmannin reduced the migration of EA.Hy926 cells (Figure 4A). It was obvious that the cell migration distance in both the wortmannin group (w group) and the control group was increased with the duration, but there was no significant difference between them. In addition, the cell migration distance of IL-8 group increased significantly compared with the other group at same time point (Figure $4 \mathrm{~B}, P<0.05$ ). Specifically, the cell migration distance of $\mathrm{w}+\mathrm{IL}-8$ group was significantly decreased compared to IL- 8 group from $2 \mathrm{~h}$ to $6 \mathrm{~h}$ (Figure $4 \mathrm{~B}, P<0.01$ ), suggesting that $\mathrm{PI} 3 \mathrm{~K}$ is involved in IL-8 induced cell migration.

\section{PI3K inhibitor inhibits IL-8 induced cytoskeleton changes}

Figure 5 showed that wortmannin induced an increase in filopodia formation, but led to decreased membrane ruffling and reduced formation of the actin stress fiber. In 5 min group and 10 min group, filopodia could be observed. Cells did not show actin accumulation at the leading edge lamellipodia in $30 \mathrm{~min}$ group. Wortmannin treatment abrogated IL-8 induced rearrangements of actin filaments in $1 \mathrm{~h}, 2 \mathrm{~h}, 4 \mathrm{~h}$ and $6 \mathrm{~h}$ group. Taken together, these results suggest that wortmannin pretreatment prevents IL-8 induced actin filaments rearrangements and PI3K signaling is required for the cytoskeletal changes stimulated by IL-8.

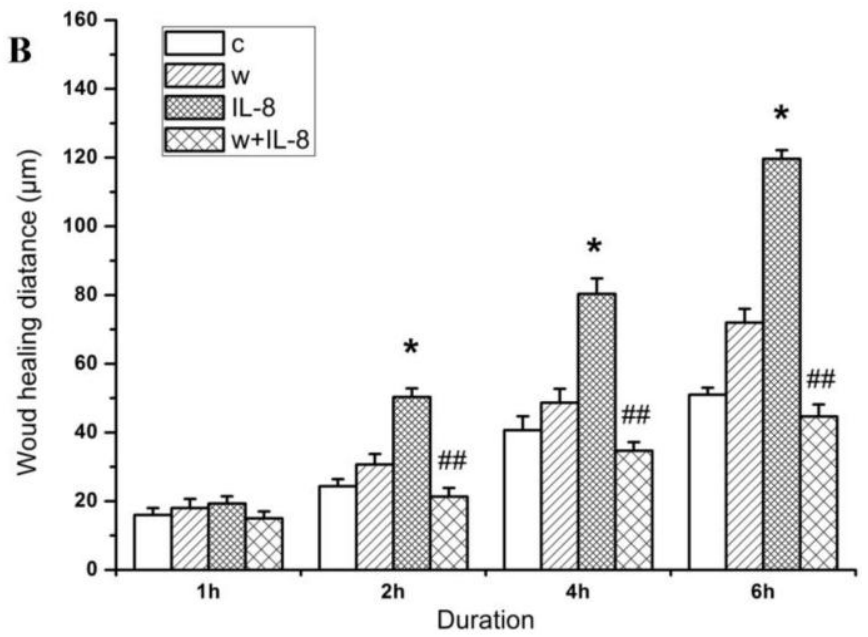

FIG.4. Wortmannin inhibits the cell migration induced by IL-8. (A) After 20 min pretreatment with 100 mM wortmannin, EA.Hy926 cells were subjected to a migration assay in the absence or presence of $100 \mathrm{ng} / \mathrm{ml} \mathrm{IL-8.} \mathrm{The} \mathrm{cell}$ migration was increased along with the stimulated time. (B) The changes of the cell migration distance in different groups with the duration. IL-8 group. ${ }^{*} P<0.05$ vs. other groups; ${ }^{\#} P<0.01$ vs. IL-8 group. Scale bar $=600 \mu \mathrm{m}$. Three independent experiments were conducted. 

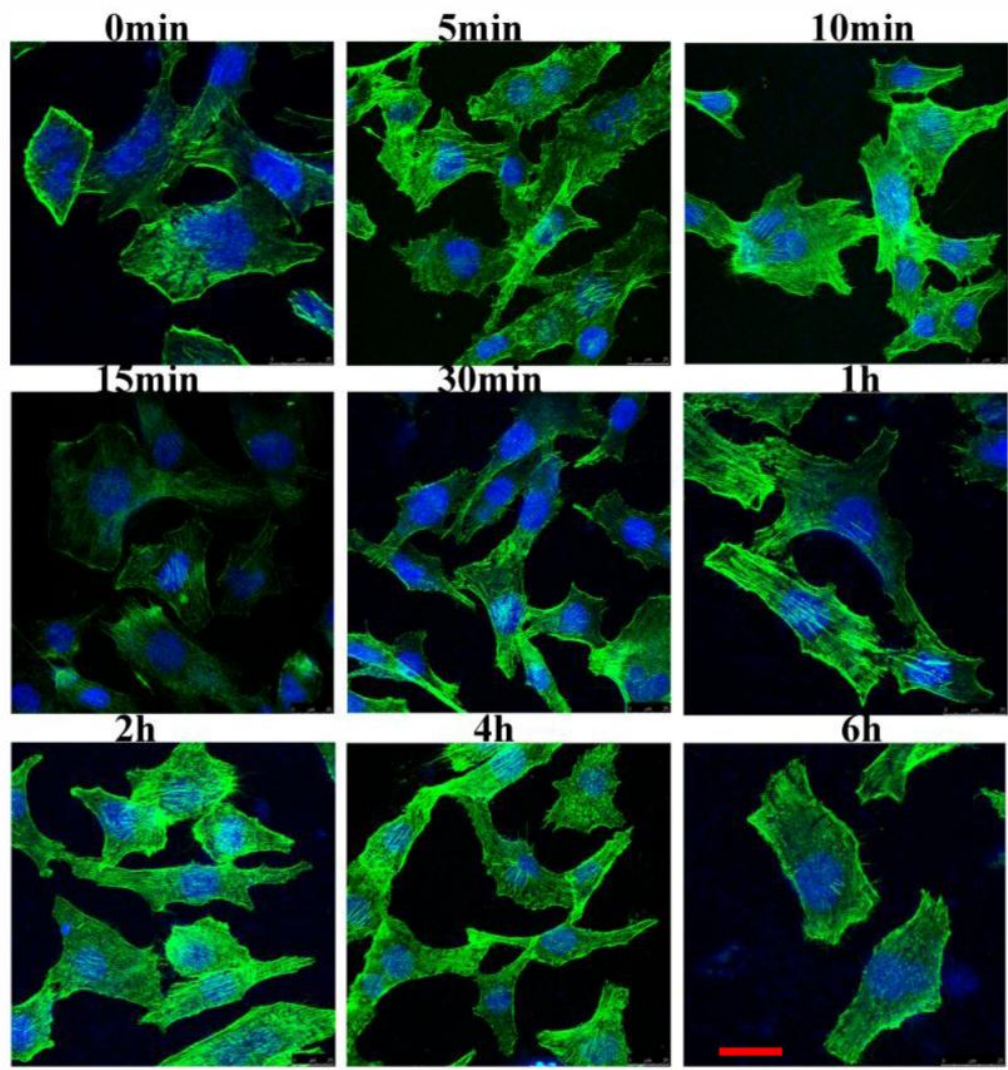

FIG.5. Wortmannin inhibits IL-8-induced cytoskeleton changes. After 20 min pretreatment with $100 \mathrm{mM}$ wortmannin, EA.Hy926 cells were treated as described in Fig.2. In the $5 \mathrm{~min}$ and $10 \mathrm{~min}$ groups, filopodia could be observed. Cells did not show actin accumulation at the leading edge lamellipodia in 30 min group. In $1 \mathrm{~h}, 2 \mathrm{~h}, 4 \mathrm{~h}$ and $6 \mathrm{~h}$ groups, wortmannin treatment abrogated IL-8 induced rearrangements of actin filaments. Scale bar $=25 \mu \mathrm{m}$. Shown were representative images of an interphase cell.

\section{PI3K inhibitor inhibits IL-8 induced upregulation of Rac1 and RhoA in the endothelial cells}

We stimulated EA.Hy926 cells with IL-8 in the presence or absence of wortmannin and examined the expression of Rac1 and RhoA (Figure 6). When cells were pretreated with wortmannin alone, RhoA expression showed no significant change after the cells were treated by up to $2 \mathrm{~h}$. However, in $4 \mathrm{~h}$ and $6 \mathrm{~h}$ groups, RhoA expression increased significantly compared to control group, indicating that the wortmannin mediated inhibition of RhoA expression might be reversed after $4 \mathrm{~h}$ (Figure 6A, $P<0.05$ ). In contrast, Rac1 expression in all wortmannin pretreated groups decreased significantly compared to control group $(P<0.01)$ (Figure $6 \mathrm{~B})$. These results suggest that PI3K is implicated in Rac1 expression. Compare to Rac1, RhoA seems less sensitive to wortmannin.

When cells were pretreated with wortmannin and then stimulated by IL-8, RhoA expression decreased at $5 \mathrm{~min}$ (Figure 6C, $P<0.05$ ), $30 \mathrm{~min}, 1 \mathrm{~h}$ and 2 h (Figure 6C, $P<0.01$ ) compared to IL-8 stimulation alone at the same time points. On the other hand, as shown in Figure 6D, Rac1 expression induced by IL-8 was notably inhibited by wortmannin at the $5 \mathrm{~min}, 2$ $\mathrm{h}$, and $6 \mathrm{~h}$ time points $(P<0.01)$. Collectively, these results suggest that IL-8 induced upregulation of Rac1 and RhoA in the endothelial cells is at least partially mediated by PI3K signaling. 

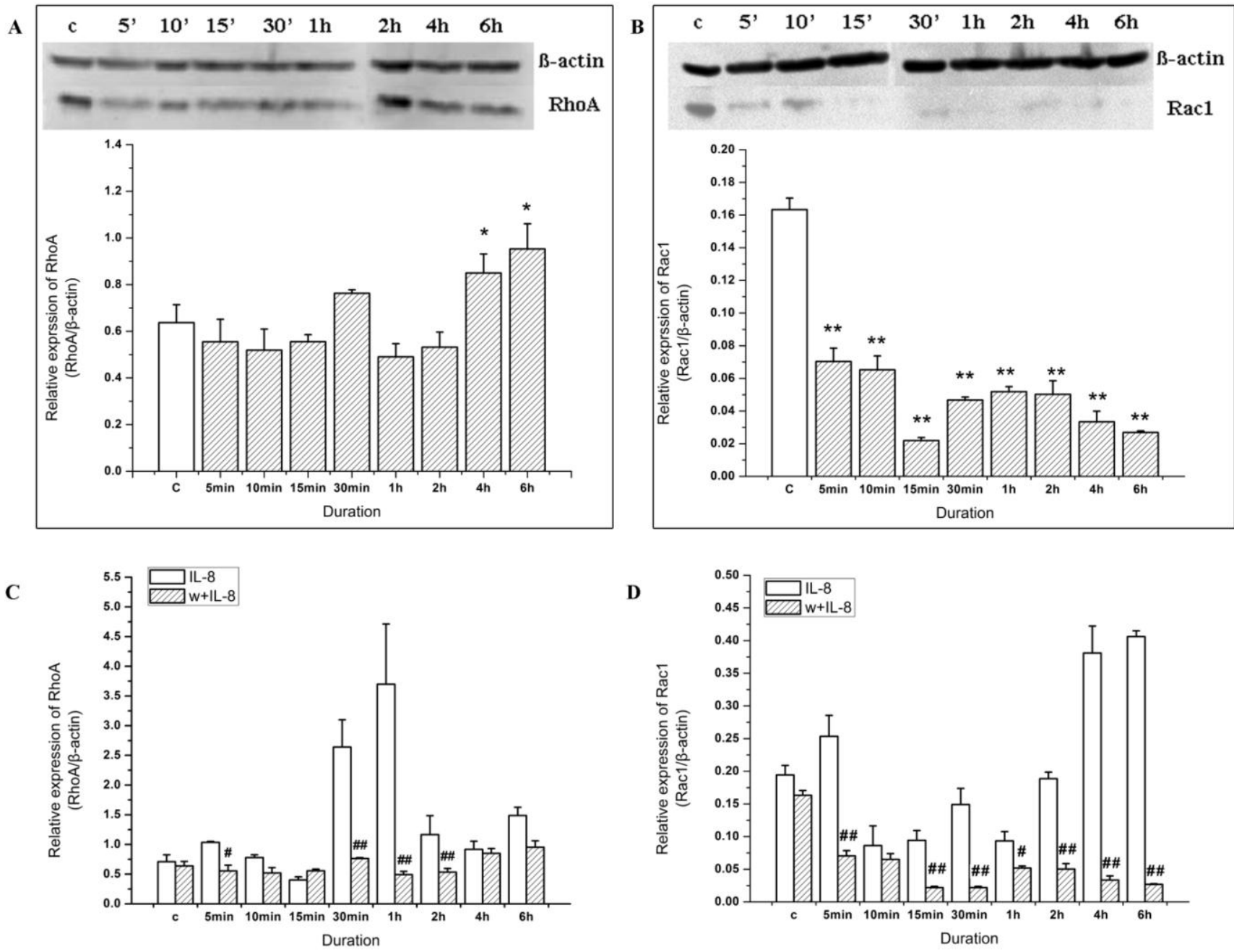

FIG.6. Wortmannin inhibits the upregulation of Rac1 and RhoA induced by IL-8. After 20 min pretreatment with 100 $\mathrm{mM}$ wortmannin, the level of total RhoA in EA. Hy926 cells was determined by Western blotting (A). ${ }^{*} P<0.05 \mathrm{vs}$. control group; and the level of total Rac1 in EA.Hy926 cells was determined by Western blotting (B). ${ }^{* *} P<0.01$ vs. control group. After 20 min pretreatment with $100 \mathrm{mM}$ wortmannin and stimulated by IL-8 for the indicated time, the level of total RhoA in EA.Hy926 cells was determined by Western blotting (C) \# $P<0.05$; ${ }^{\# \#} P<0.01$; and the level of total Rac1 in EA.Hy926 cells was determined by Western blotting (D) ${ }^{\#} P<0.05$; ${ }^{\# \#} P<0.01$.

\section{Discussion}

Cell migration is usually initiated in response to extracellular cues, including diffusible factors, signals on neighboring cells, and/or signals from the extracellular matrix [9]. Our previous study demonstrated that IL-8 could induce endothelial cell migration. Specifically, cell motility was induced by IL-8 significantly at a concentration of $100 \mathrm{ng} / \mathrm{ml}$ [4]. However, the mechanism by which IL-8 induces cell migration remains elusive. It is well known that cell migration is closely related to cytoskeleton. The remodeling of actin cytoskeleton includes filopodia, lamellipodia, and stress fibers. All the three structures are essential to drive the several steps of actin-based endothelial cell motility [10]. Schraufstatter et al. showed that IL-8 activated both the CXCR1 and the CXCR2 (two IL-8 receptors). Activation of the CXCR1 was transient and characterized by stress fiber formation, whereas activation of CXCR2 was prolonged and associated with cell contraction [11].

In the present study, we found that IL-8 treatment not only induced the membrane ruffles and lamellipodia extensions, but also increased the actin stress fibers. Compared to other cytokines, the cytoskeleton change induced by IL-8 lasted for a long time. Especially, the stress fiber maintained for about $5 \mathrm{~h}$, which indicated that the effect of IL-8 on stress fiber was relatively persistent.

Rac1 induces the assembly of a meshwork of actin filaments at the cell periphery to produce lamel- 
lipodia and membrane ruffles. Our results showed that when endothelial cells were stimulated by IL- 8 for $5 \mathrm{~min}$, Rac1 expression increased, accompanied by the formation of membrane ruffles. Therefore, RhoA expression at this time point may play a role in the membrane ruffles formation. Interestingly, in our study, lamellipodia at the leading edge was observed in the cells after IL-8 stimulation for $15 \mathrm{~min}$ or $30 \mathrm{~min}$, but the expression of Rac1 decreased compared to unstimulated control group, suggesting that RhoA may participate in the lamellipodia formation in cell migration stimulated by IL-8. Based on these results, we conclude that IL-8 could quickly upregulate Rac1 expression within $5 \mathrm{~min}$ to induce membrane ruffles, while the formation of stress fibers is associated with the upregulate of Rac1 and RhoA expression in cells treated for longer time, i.e. $1 \mathrm{~h}$ to $6 \mathrm{~h}$.

Interestingly, the expression of RhoA was more abundant than Rac1 both in the control group and experimental groups. However, the results from the transient transfection showed that dominant negative forms of RhoA and Rac1 could both decrease the cell migration induced by IL-8 remarkably. In addition, dominant active forms of Rac1 or RhoA alone significantly enhanced the cell migration. Thus we conclude that both RhoA and Rac1 take part in IL-8 induced cell migration, since interference with either pathway strongly impaired these responses. Taken together, we postulate that the relative quantity of RhoA and Rac1 might have no relationship with their function in the cell migration induced by IL-8, and both Rac1 and RhoA play important roles in IL-8 induced migration of endothelial cells.

PI3K is one of the most important regulatory proteins involved in controlling key cellular functions, such as cell growth, aging, transformation and cell migration [3]. Activation of the PI3K p110 subunit in breast cancer cells has been reported to be necessary for EGF induced actin nucleation, an important cytoskeleton change for cell migration. Huang et al. reported that PI3K played an important role in EGF induced cell migration in MDA-MB-231 cells [12]. Moreover, PI3K could activate Rac1 at the leading edge which was activated either via tyrosine kinases or via G-protein-coupled receptors [13]. PI3K inhibitor wortmanin and dominant-negative PI3K constructs could inhibit the membrane ruffling by Rac-dependent pathway which was stimulated by platelet-derived growth factor (PDGF) and insulin. This inhibition would be override by overexpression of wild type or constitutively active Rac. Therefore, PI3K might be upstream of Rac activation in cells stimulated by IL- 8 .
In this study, we firstly confirmed the critical role of PI3K in IL-8 induced cell migration by demonstrating that wortmannin could obviously inhibit the cell migration induced by IL- 8 . The changes of cytoskeleton showed that pretreatment by wortmannin would reduce the membrane ruffling and stress fibers significantly. These observations suggest that PI3K signaling is required for these cytoskeletal changes. Furthermore, by Western blot analysis we found that wortmannin led to decreased expression of Rac1 and RhoA in cells treated by IL-8. Collectively, these data suggest that PI3K functions as an upstream activator of Rac1 and RhoA and subsequently affects the cytoskeleton and induces endothelial cell migration.

In summary, our data demonstrate that IL-8-PI3K-Rac1/RhoA signaling pathway plays a vital role in IL-8 induced endothelial cell migration. Further analysis of this signaling cascade will provides new insight into the molecular mechanisms by which IL-8 contributes to tumor angiogenesis and metastasis.

\section{Acknowledgments}

This study was supported in part by grant from National Natural Science Foundation of China (No. 30570450, 10772127, 10972148), Sichuan Youth Science \& Technology Foundation (No. 06ZQ026-009), Program for New Century Excellent Talents in University of China (No. NCET-06-0791), and China Medical Board (Project number: 82-412).

\section{Conflict of Interests}

The authors have declared that no conflict of interest exists.

\section{References}

1. Yoshimura T., Matsushima K, Oppenheim JJ, et al. Neutrophil chemotactic factor produced by lipopolysaccharide (LPS)-stimulated human blood mononuclear leukocytes: partial characterization and separation from interleukin 1 (IL 1). J Immunol. 1987;139 (3):788-93

2. Ridley AJ. Rho GTPases and cell migration. Journal of Cell Science.2001;114 (15):2713-2722

3. Cain RJ and Ridley AJ. Phosphoinositide 3-kinases in cell migration. Biology of the Cell. 2009;101 (1):13-29

4. Zou MJ, Liu XH, Li Y, Lai Y. Experimental study on the migration of vascular endothelial cells stimulated by IL-8. J Biomed Eng. 2006;23 (5):1013-1016

5. Lai Y, Liu XH, Wu J, Zeng Y, Yue A, He XL. Rac1 mediates the Migration of Endothelial cell Induced by IL-8. J Biomed Eng 2008;25 (2):357-362

6. Edgell C, McDonald C and Graham J. Permanent cell line expressing human factor VIII-related antigen established by hybridization. Proceedings of the National Academy of Sciences of the United States of America. 1983;80 (12):3734

7. Schmitt CA, Handler N, Heiss EH, et al. No evidence for modulation of endothelial nitric oxide synthase by the olive oil 
polyphenol hydroxytyrosol in human endothelial cells. Atherosclerosis. 2007;195 (1):e58-64

8. Subauste MC, Von Herrath M, Benard V, Chamberlain CE, Chuang TH, Chu K, Bokoch GM, Hahn KM. Rho Family Proteins Modulate Rapid Apoptosis Induced by Cytotoxic $\mathrm{T}$ Lymphocytes and Fas. J Biol Chem. 2000;275(13):9725-33.

9. Ridley AJ, Schwartz MA, Burridge K, Firtel RA, Ginsberg MH, Borisy G, Parsons JT, Horwitz AR. Cell migration: Integrating signals from front to back. Science. 2003;302 (5651):1704-1709

10. Lamalice L, Le Boeuf F, and Huot J. Endothelial cell migration during angiogenesis. Circulation Research. 2007;100 (6):782

11. Schraufstatter IU., Chung J and Burger M. IL-8 activates endothelial cell CXCR1 and CXCR2 through Rho and Rac signaling pathways. Am J Physiol Lung Cell Mol Physiol. 2001;280 (6):L1094-103

12. Huang Q, Shen HM, and Ong CN. Emodin inhibits tumor cell migration through suppression of the phosphatidylinositol 3-kinase-Cdc42/Rac1 pathway. Cellular and Molecular Life Sciences. 2005;62 (10):1167-1175

13. Welch HC, Coadwell WJ, Stephens LR, Hawkins PT. Phosphoinositide 3-kinase-dependent activation of Rac. Febs Letters. 2003;546 (1):93-97

14. Yearley JH, Xia D, Pearson CB, et al. Interleukin-18 predicts atherosclerosis progression in SIV-infected and uninfected rhesus monkeys (Macaca mulatta) on a high-fat/high-cholesterol diet. Lab Invest. 2009;89 (6):657-67 